ARTICLE

DOI: $10.1038 / s 41467-018-05632-4$

\title{
Charge-tuneable biexciton complexes in monolayer $\mathrm{WSe}_{2}$
}

Matteo Barbone ${ }^{1,2}$, Alejandro R.-P. Montblanch', Dhiren M. Kara ${ }^{1}$, Carmen Palacios-Berraquero', Alisson R. Cadore ${ }^{2}$, Domenico De Fazio², Benjamin Pingault ${ }^{1}$, Elaheh Mostaani ${ }^{2}, \mathrm{Han} \mathrm{Li}^{3}$, Bin $\mathrm{Chen}^{3}$, Kenji Watanabe (i) ${ }^{4}$, Takashi Taniguchi ${ }^{4}$, Sefaattin Tongay ${ }^{3}$, Gang Wang${ }^{2}$, Andrea C. Ferrari ${ }^{2}$ \& Mete Atatüre ${ }^{1}$

Monolayer transition metal dichalcogenides have strong Coulomb-mediated many-body interactions. Theoretical studies have predicted the existence of numerous multi-particle excitonic states. Two-particle excitons and three-particle trions have been identified by their optical signatures. However, more complex states such as biexcitons have been elusive due to limited spectral quality of the optical emission. Here, we report direct evidence of two biexciton complexes in monolayer tungsten diselenide: the four-particle neutral biexciton and the five-particle negatively charged biexciton. We distinguish these states by powerdependent photoluminescence and demonstrate full electrical switching between them. We determine the band states of the elementary particles comprising the biexcitons through magneto-optical spectroscopy. We also resolve a splitting of $2.5 \mathrm{meV}$ for the neutral biexciton, which we attribute to the fine structure, providing reference for subsequent studies. Our results unveil the nature of multi-exciton complexes in transitionmetal dichalcogenides and offer direct routes towards deterministic control in many-body quantum phenomena.

\footnotetext{
${ }^{1}$ Cavendish Laboratory, University of Cambridge, JJ Thomson Ave., Cambridge CB3 OHE, UK. ${ }^{2}$ Cambridge Graphene Centre, University of Cambridge, Cambridge CB3 OFA, UK. ${ }^{3}$ School for Engineering of Matter, Transport and Energy, Arizona State University, Tempe, AZ 85287, USA. ${ }^{4}$ National Institute for Materials Science, Tsukuba, Ibaraki 305-0034, Japan. These authors contributed equally: Matteo Barbone, Alejandro R.-P. Montblanch. Correspondence and requests for materials should be addressed to A.C.F. (email: acf26@cam.ac.uk) or to M.Aür. (email: ma424@cam.ac.uk)
} 
n monolayer (1L) transitionmetal dichalcogenides (TMDs), the three-atom thickness of the material reduces the dielectric screening with respect to their bulk counterparts ${ }^{1,2}$. As a result of this and of their large effective mass, excitons (quasi-particle states formed of electrons and holes via Coulomb interaction) have binding energies of hundreds of $\mathrm{meV}^{1,2}$ and are stable at room temperature. The physics of light-matter interaction is also enriched by two inequivalent valleys having opposite spin-locked valley indices $^{3}$ at the $\mathrm{K}$ points of the Brillouin zone, in which radiative recombination generates photons carrying opposite angular momenta ${ }^{4,5}$. These properties motivated the exploration of exciton and polariton ${ }^{6}$ condensation $^{7,8}$ and superfluidity ${ }^{9}$, and the exploitation of the spin and valley degrees of freedom as means to carry and manipulate information in quantum optoelectronic devices ${ }^{3,10}$. In the limit of quantum-confined excitons, the presence of localised single-photon emitters that can be induced deterministically ${ }^{11,12}$ and generated by electroluminescence ${ }^{13}$, makes TMDs a promising platform for the field of quantum photonics. Contrary to the exciton $^{14,15}$ and trion ${ }^{16,17}$ states, optical studies of biexciton complexes $^{18,19}$ in 1L-TMDs have been challenging ${ }^{20-26}$ : inhomogeneous broadening ${ }^{27}$ and defect bands ${ }^{28}$ have limited their unambiguous identification and control. As a consequence, previous experimental findings ${ }^{20-23,25,26}$ assigned neutral biexcitons a larger binding energy than trions, in contrast to theoretical predictions ${ }^{29-33}$, whereas ref. ${ }^{24}$ observed a peak in 1L-molybdenum diselenide $\left(\mathrm{MoSe}_{2}\right)$ in the expected energy range, which they labelled as the neutral biexciton.

Here, we use continuous wave photoluminescence (PL) measurements at cryogenic temperature combined with electrical gating and magnetic field to identify the four-particle neutral biexciton $\left(\mathrm{XX}^{0}\right)$ and the five-particle negatively charged biexciton, the quinton ${ }^{29}\left(\mathrm{XX}^{-}\right)$in 1L-tungsten diselenide $\left(\mathrm{WSe}_{2}\right)$. We also observe a splitting in $\mathrm{XX}^{0}$, which we attribute to its fine structure. Our results demonstrate tuneable access to multi-exciton complexes in TMDs and provide new ways to study and control multi-exciton phenomena.

\section{Results}

Design and optical characterisation of heterostructures. We use recent advances in material and device processing 27,34 to suppress the effects that degrade the optical quality of $1 \mathrm{~L}-\mathrm{WS} e_{2}$. To reduce the PL spectral linewidths ${ }^{27}$ we place a layered material heterostructure $(\mathrm{LMH})$ formed of $1 \mathrm{~L}-\mathrm{WSe}_{2}$ encapsulated between two flakes of multilayer hexagonal boron nitride (ML-hBN) on a $\mathrm{Si} /$ $\mathrm{SiO}_{2}$ substrate. To suppress the effect of $\mathrm{SiO}_{2}$ charge traps we place a few-layer graphene (FLG) crystal below the bottom MLhBN. The inset of Fig. 1a shows a schematic of the LMH (see
Methods, and Supplementary Notes 1 and 2 for sample preparation and characterisation).

We illuminate the $\mathrm{LMH}$ with continuous laser excitation at 658 $\mathrm{nm}$ and collect its optical emission at $4 \mathrm{~K}$ (see Methods for further details on the optical measurements): Fig. 1a is a representative PL spectrum. Consistent with previous reports, we identify the bright neutral exciton ${ }^{10}, \mathrm{X}^{0}$, at $\sim 1.728 \mathrm{eV}$ (width $\sim 5 \mathrm{meV}$ ), the negatively charged intervalley $\operatorname{trion}^{35}, \mathrm{X}^{-}$inter, at $\sim 1.699 \mathrm{eV}$, the negatively charged intravalley trion ${ }^{35}, \mathrm{X}^{-}{ }_{\text {intra }}$, at $\sim 1.692 \mathrm{eV}$, and the dark neutral exciton ${ }^{36,37}, \mathrm{X}_{\text {dark }}^{0}$, at $\sim 1.685 \mathrm{eV}$. Here, bright refers to excitons with in-plane dipole and spin-allowed radiative recombination $2,36,37$, whereas dark refers to excitons with outof-plane dipole and spin-forbidden radiative recombination ${ }^{2,36,37}$, for which emission only occurs in plane but is captured partially by our high numerical aperture objective. The peak at $\sim 1.711 \mathrm{eV}$, $\sim 4 \mathrm{meV}$ wide, is a good candidate for $\mathrm{XX}^{0}$, as it appears in the theoretically predicted energy range ${ }^{29-32}$. The peak at $\sim 1.679 \mathrm{eV}$, $\sim 6 \mathrm{meV}$ wide, was previously labelled as neutral biexciton emission $^{20}$, although it appears in the energy range predicted ${ }^{29,31}$ for $\mathrm{XX}^{-}$. In the top part of Fig. 1a, we include the emission energies of single- and multi-exciton species in $\mathrm{ML}^{-\mathrm{WSe}_{2}}$ calculated via diffusion Quantum Monte Carlo ${ }^{29}$ combined with environment screening (See Methods for details).

Unveiling the presence and nature of biexcitons. Figure $1 \mathrm{~b}$ displays the PL intensity $I$, defined as peak area, as a function of excitation power $P$ (with $\left.I \propto P^{\alpha}\right)$ for $\mathrm{X}^{0}$ (filled black circles), $\mathrm{XX}^{0}$ (filled red circles) and $\mathrm{XX}^{-}$(filled blue circles). For reference, we plot solid curves corresponding to a linear $(\alpha=1$, black) and quadratic ( $\alpha=2$, red) behaviour. We expect superlinear behaviour for biexcitons reaching $\alpha=2$ in thermodynamic equilibrium $^{18,19}$. The power dependence of $\mathrm{XX}^{0}$ follows the quadratic curve, while that of $\mathrm{XX}^{-}$is superlinear with fitted $\alpha \sim 1.55 \pm 0.03$ (dashed blue curve). Both trends of $\mathrm{XX}^{0}$ and $\mathrm{XX}^{-}$are therefore consistent with a biexcitonic origin and contrast the linear behaviour of $\mathrm{X}^{0}$. The deviation of $\mathrm{XX}^{-}$from $\alpha=2$ possibly stems from the competition of electron capture from other optically induced excitons. Remaining peaks of Fig. 1a follow an approximately linear power dependence.

To differentiate the charged and neutral biexciton $\mathrm{XX}^{0}$ and $\mathrm{XX}^{-}$, we fabricate a charge-tuneable device starting from a new $\mathrm{LMH}$ analogous to the first one but with the addition of one electrode to the FLG and of a second electrode to an uncovered $1 \mathrm{~L}-\mathrm{WSe}_{2}$ portion (see Methods). Figure $2 \mathrm{a}, \mathrm{b}$ shows the schematic and the optical image of the device, respectively. Figure $2 \mathrm{c}$ displays how the PL spectrum is modified as a function of voltage $V$. The charging regime modifies the optical signatures of
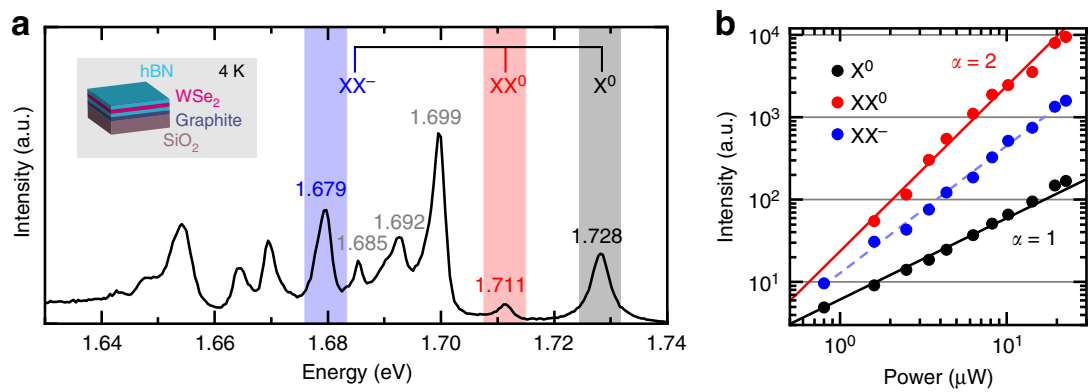

Fig. $1 \mathrm{PL}$ spectrum and power dependence of encapsulated 1L-WSe 2 at $4 \mathrm{~K}$. a PL spectrum (black curve, linear scale) of encapsulated $1 \mathrm{~L}-\mathrm{WS} \mathrm{e}_{2}$. Excitation wavelength: $658 \mathrm{~nm}$. The top part of the figure lists the calculated spectral locations of $X^{0}$ (grey), $X^{0}$ (red) and $X^{-}$(blue) in the presence of a screening environment. $\mathbf{b}$ Double logarithmic plot of PL intensity as a function of excitation power for $X^{0}$ (black filled circles), $X^{0}$ (red filled circles) and $X X^{-}$(blue filled circles). The solid curves represent $I \propto P^{\alpha}$ for a quadratic ( $\alpha=2$, red) and linear ( $\alpha=1$, black) behaviour. The dashed blue curve is a fit to PL intensity, yielding an $\alpha$ of 1.55 . For clarity of display, we multiply $X X^{0}$ by 4 and $X^{0}$ by 0.4 

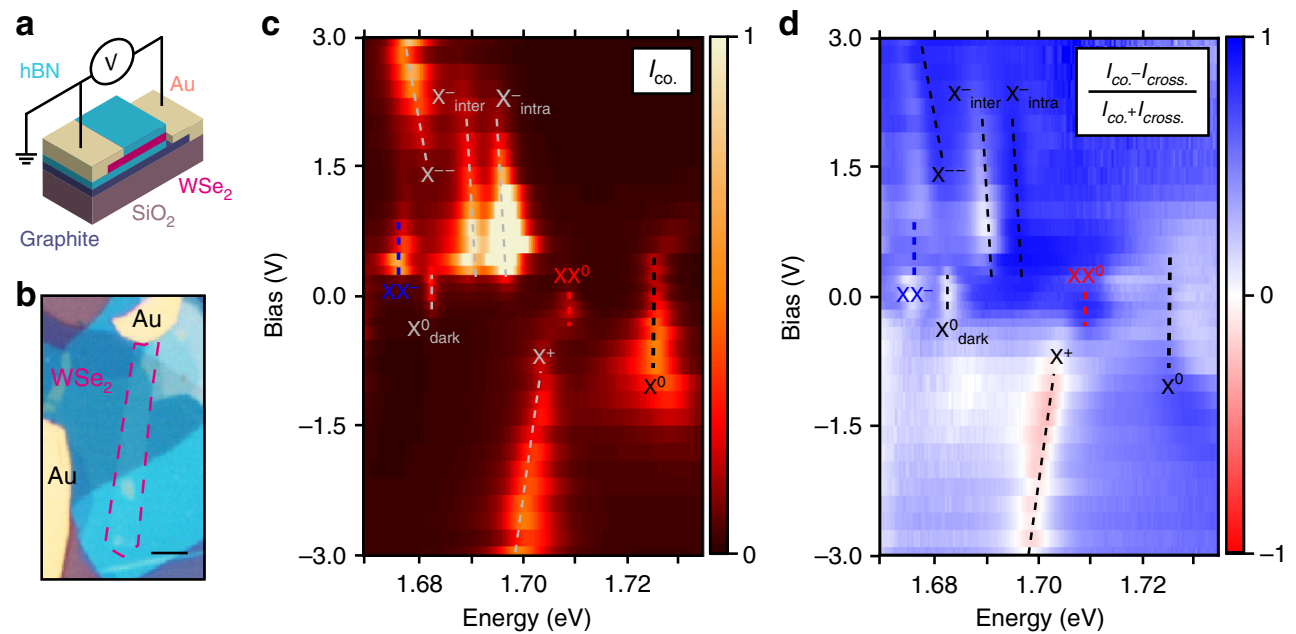

Fig. 2 Charge dependence of PL. a Schematic and $\mathbf{b}$ optical image of the charge-tuneable device. The red dashed frame highlights the $1 \mathrm{~L}-\mathrm{WSe} \mathrm{flake}_{2}$. The scale bar is $5 \mu \mathrm{m}$. c Circular co-polarised PL intensity $\left(I_{\sigma+/ \sigma+}+I_{\sigma-/ \sigma-}\right)$ as a function of applied bias. The dashed lines are a guide to the eye to highlight each

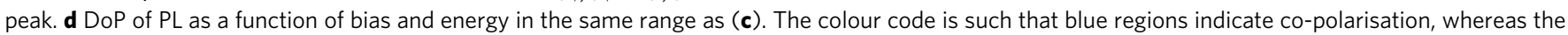
red regions indicate counter-polarisation with respect to excitation polarisation

$1 \mathrm{~L}-\mathrm{WSe}_{2}$ strongly. The presence of $\mathrm{X}^{0}$ and $\mathrm{X}_{\text {dark }}$ at $V \sim 0 \mathrm{~V}$ shows that the material has a negligible intrinsic charge doping. At the same bias, Fig. $2 \mathrm{c}$ also shows emission from $\mathrm{XX}^{0}$. In the electron-charged regime $(V>0)$ fluorescence from $\mathrm{X}^{0}, \mathrm{XX}^{0}$ and $\mathrm{X}^{0}{ }_{\text {dark }}$ vanishes, while emission from $\mathrm{X}^{-}{ }_{\text {inter }}, \mathrm{X}^{-}{ }_{\text {intra }}$ and $\mathrm{XX}^{-}$arises. Around $2 \mathrm{~V}$ the $\mathrm{X}^{-}$emission switches to a new peak at $\sim 1.681 \mathrm{eV}$, likely the next charging state of the trion, $\mathrm{X}^{--}$. This peak was previously assigned to the fine structure of $\mathrm{X}^{-}$in experiments on bare material ${ }^{10}$. Negative bias is the hole-charged regime, where only $\mathrm{X}^{0}$ and the positively charged trion $\mathrm{X}^{+}$are visible (refs. ${ }^{10,35}$ ). The voltage-dependence of our PL measurements thus clarifies the difference between the two biexciton species: the presence of $\mathrm{XX}^{0}$ only at charge neutrality confirms this is the charge-neutral biexciton, and the appearance of $\mathrm{XX}^{-}$ only in the electron-charged regime proves it to be the negatively charged biexciton.

We then analyse the correlation between excitation and emission polarisations in the different charging regimes (Fig. 2d). We plot the degree of circular polarisation $\left[\mathrm{DoP}=\left(I_{\text {co. }}-I_{\text {cross. }}\right) /\right.$ $\left.\left(I_{\text {co. }}+I_{\text {cross. }}\right)^{4}\right]$ where $I_{\text {co. }}\left(I_{\text {cross. }}\right)$ is the intensity of the circularly polarised light with the same (opposite) helicity in the excitation and detection paths. We refer to the two orthogonal helicities as $\sigma^{-}$and $\sigma^{+}$. At $0 \mathrm{~V}, \mathrm{XX}^{0}$ has $\mathrm{DoP}>80 \%$, while $\mathrm{X}^{0}$ dark shows no circular polarisation $^{36,37}$, as expected. At $0.8 \mathrm{~V}, \mathrm{X}^{-}$inter has DoP > $90 \%, \mathrm{X}^{-}$intra has $\mathrm{DoP}<10 \%$ and $\mathrm{XX}^{-}$has DoP $\sim 55 \%$. The circular polarisation of photons from both $\mathrm{XX}^{0}$ and $\mathrm{XX}^{-}$thus implies that dissociation occurs with the recombination of a bright exciton, as a dark exciton would emit linearly polarised light ${ }^{36,37}$. The DoP of $\mathrm{XX}^{-}$is close to the average of the DoP of $\mathrm{X}^{-}$inter and $\mathrm{X}^{-}$intra, suggesting that the recombination mechanisms of both $\mathrm{X}^{-}$inter and $\mathrm{X}^{-}$intra contribute ${ }^{38}$ to that of $\mathrm{XX}^{-}$.

Behaviour of exciton complexes in magnetic field. The electrons and holes comprising the biexcitons can occupy multiple combinations of band states. To identify them, we resort to the variation of PL as a function of an out-of-plane magnetic field. Figure $3 \mathrm{a}$ shows the $\sigma^{-}$polarised PL of $\mathrm{X}^{0}$ and $\mathrm{XX}^{0}$ under copolarised $\left(\sigma^{-}\right)$excitation. We resolve a finite splitting in the $\mathrm{XX}^{0}$ emission, with a separation of $2.5 \mathrm{meV}$ between the two peaks labelled $\mathrm{XX}^{0}{ }_{1}$ and $\mathrm{XX}^{0}{ }_{2}$ (line-cut spectra at different magnetic fields are shown in Supplementary Fig. 3). Figure $3 \mathrm{~b}$ shows the $\sigma^{+}$ polarised PL of $\mathrm{X}^{0}$ and $\mathrm{XX}^{0}$ under cross-polarised $\left(\sigma^{-}\right)$excitation. Here, only $\mathrm{XX}^{0}{ }_{2}$ remains visible, revealing a different DoP for $\mathrm{XX}^{0}{ }_{1}$ and $\mathrm{XX}^{0}{ }_{2}$, in analogy to the different DoP between $\mathrm{X}^{-}$inter and $\mathrm{X}^{-}$intra.

The energy of the splitting excludes one of the peaks to be a phonon replica, and the two peaks reveal different DoP, thus we assign this doublet to fine structure introduced by exchange interaction, in analogy to the case of the splitting between $\mathrm{X}^{-}$inter and $\mathrm{X}^{-}{ }_{\text {intra }}{ }^{39}$. This experimental observation of the $\mathrm{XX}^{0}$ fine structure will set a reference for further computational studies, which otherwise suffer from limitations due to the complex treatment of the exchange interaction. Additionally, the PL intensity of $\mathrm{XX}^{0}$ emission increases when it shifts to higher energies, in contrast to that of $\mathrm{X}^{0}$. We observe the same behaviour for $\mathrm{XX}^{-}$in Fig. 3c, where the co-polarised $\mathrm{PL}$ from the recombination of the quasi-particle also shows valley-dependent Zeeman shift.

In Fig. $3 \mathrm{~d}$ we plot the energies of $\mathrm{X}^{0}, \mathrm{XX}^{0}{ }_{1}, \mathrm{XX}^{0}{ }_{2}$ and $\mathrm{XX}^{-}$as a function of magnetic field. For each multi-exciton species, we calculate the Landé factor $g$, defined as $\Delta E=g \mu_{\mathrm{B}} B$, where $\Delta E=$ $E_{\sigma+}-E_{\sigma-}$ is the difference in the emission energy of excitons in opposite valleys, $\mu_{\mathrm{B}}=e \hbar / 2 m_{e}=58 \mu \mathrm{eV} \mathrm{T}^{-1}$ is the Bohr magneton and $B$ is the magnetic field. We derive $g \sim-4.44 \pm 0.12$ for $\mathrm{X}^{0}$ consistent with previous observations ${ }^{40}, \sim-4.10 \pm 0.15$ for $\mathrm{XX}^{0}$ and $\sim-3.86 \pm 0.17$ for $\mathrm{XX}^{-}$. We note that these values do not represent the total $\mathrm{g}$ factor of the multi-particle states, but rather belong to their optically active components.

The emission intensities of $\mathrm{XX}^{0}$ and $\mathrm{XX}^{-}$change dramatically with magnetic field, being stronger when shifted to higher energy. Figure 3e displays the $I_{\sigma-/ \sigma-} / I_{\sigma+/ \sigma+}$ ratio as a function of magnetic field for $\mathrm{XX}^{0}{ }_{1}+\mathrm{XX}^{0}{ }_{2}$ and $\mathrm{XX}^{-}$. For comparison, we also include $I_{\sigma-/ \sigma-} / I_{\sigma+/ \sigma+}$ for $\mathrm{X}^{0}$. At zero magnetic field $I_{\sigma-/ \sigma-} / I_{\sigma+/ \sigma+}$ is $\sim 1$ for all peaks, i.e., the two valleys have the same exciton population. When magnetic field is applied, $I_{\sigma-/ \sigma-} / I_{\sigma+/ \sigma+}$ remains unaffected for $\mathrm{X}^{0}$. This can be explained by $\mathrm{X}^{0}$ in each valley recombining before reaching thermal equilibrium. In stark contrast, $\mathrm{XX}^{0}$ and $\mathrm{XX}^{-}$display strongly anti-symmetric magnetic-field dependence: for increasing magnetic field, the lower-energy transition is weaker.

We can understand the complex behaviour of the magneticfield dependent PL through the single-particle picture of the energy bands. Figure $4 a, b, c$ illustrates the effect of $B>0$ on the 

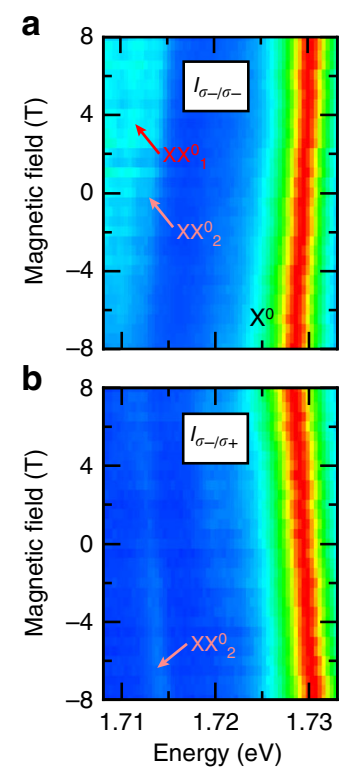
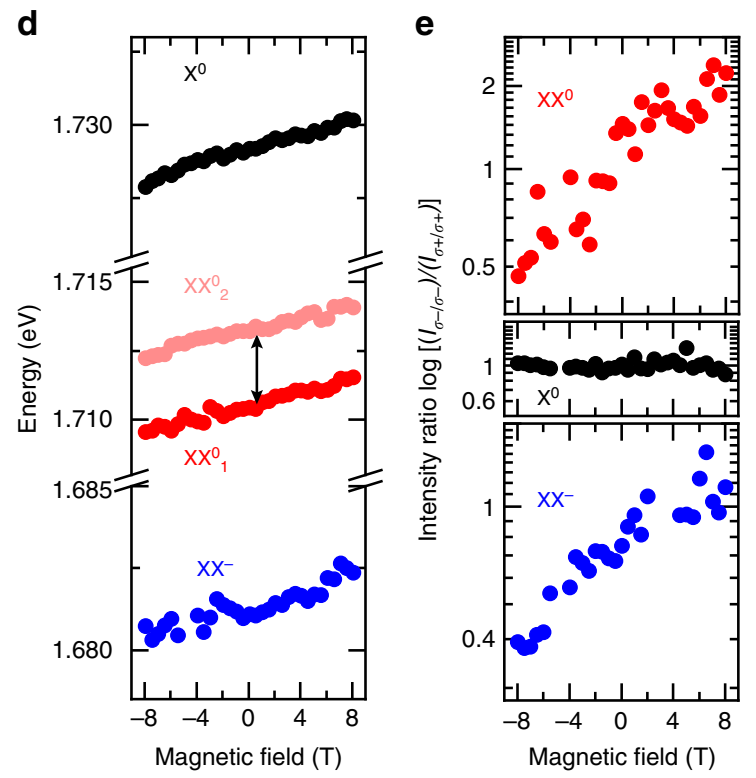

Fig. 3 Magnetic field dependence of PL. a Magnetic field dependent PL of $X^{0}$ and $X X^{0}$ in circular co-polarised and $\mathbf{b}$ cross-polarised configurations, for $\sigma^{-}$ excitation. The fine-structure lines are indicated as $X X^{0}{ }_{1}$ and $X X^{0}{ }_{2}$. The emission of $X X^{0}$ brightens with increasing emission energy. $X^{0}$ is displayed for reference. c Magnetic field dependent PL of XX- in a circular co-polarised configuration, for $\sigma^{-}$excitation. In $\mathbf{a}, \mathbf{b}$ and $\mathbf{c}$ the colour scale is linear. $\mathbf{d}$ Zeeman shift in the PL spectrum of $X^{0}$ (filled black circles), $X X^{0}$ (filled red and pink circles for the two components of the fine-structure) and $X X^{-}$(filled blue circles). The double arrow is a scale bar of $2.5 \mathrm{meV}$. e PL intensity ratio of circular co-polarisation with opposite helicity $\left.\left.I_{\sigma-/ \sigma^{-}}\right) / I_{\left(\sigma_{+} / \sigma+\right.}\right)$ for $\mathrm{X}^{0}, \mathrm{XX}^{0}{ }_{1}+$ $\mathrm{XX}_{2}$ and $\mathrm{XX}^{-}$as a function of magnetic field

band structure of $1 \mathrm{~L}-\mathrm{WSe}_{2}$ around the $\mathrm{K}$ and $\mathrm{K}^{\prime}$ points, considering the contribution of the spin, valley and atomic orbital magnetic moments. The $1 \mathrm{~L}-\mathrm{WSe}_{2}$ bandgap decreases (increases) in the $\mathrm{K}\left(\mathrm{K}^{\prime}\right)$ valley as the energies of both hole and electron experience the same spin upshift (downshift), while the hole experiences a larger orbital upshift (downshift) ${ }^{40,41}$ with respect to the electron. Further, the contribution from the valley magnetic moment results in an additional upshift (downshift) of all bands in the $\mathrm{K}\left(\mathrm{K}^{\prime}\right)$ valley ${ }^{40}$. The applied magnetic field induces unequal bright exciton populations in the two valleys (Fig. 3e). This excludes the possibility that $\mathrm{XX}^{0}$ (Fig. 4a, b) may be formed by two bright or two dark excitons, as both cases would result in equally intense radiative recombination from both $\mathrm{K}$ and $\mathrm{K}^{\prime}$ at all magnetic fields. $\mathrm{XX}^{0}$ is therefore a combination of a bright and a dark exciton. Under positive magnetic field, the bright exciton component of $\mathrm{XX}^{0}$ occupies the higher-radiative energy transition in the $K^{\prime}$ valley (Fig. $3 a, b, e$ ) due to thermalisation of the photogenerated electrons. We expect this to be allowed by a longer lifetime of $\mathrm{XX}^{0}$ compared to $\mathrm{X}^{0}$, in analogy to $\mathrm{XX}^{-}$, where the lifetime was measured to be $\sim 2-100$ times longer than single excitons ${ }^{20,25}$, and also exhibiting similar polarisation properties. In parallel, the electron of the dark component of $\mathrm{XX}^{0}$ can be either in the opposite (Fig. 4a) or in the same (Fig. 4b) valley as the bright exciton component, yielding an energy shift between these two configurations, which is the origin of the fine-structure of $\mathrm{XX}^{0}$ observed in Fig. 3d.

Figure $4 \mathrm{c}$ illustrates the single-particle configuration of $\mathrm{XX}^{-}$. As for $\mathrm{XX}^{0}$, the combination of two bright excitons is excluded due to different recombination intensities in $\mathrm{K}$ and $\mathrm{K}^{\prime}$. From the similar $g$ of $\mathrm{XX}^{0}$ and $\mathrm{XX}^{-}$, we can understand this five-particle complex as a bound state of a bright exciton with a dark trion, or a bright trion with a dark exciton. Both configurations would show inequivalent valley population as for $\mathrm{XX}^{0}$ in Fig. $3 \mathrm{e}$.

Figure $4 \mathrm{~d}$ is a qualitative many-body picture for $\mathrm{XX}^{0}$ formed by a bright and a dark exciton component in opposite valleys under magnetic field. As its total Zeeman splitting depends on both the bright and the dark component, $\mathrm{XX}^{0}$ splits with a reversed energy order compared to its bright exciton component and dissociates into a dark exciton and a photon due to the dark exciton having larger $g$ than $\mathrm{X}^{0}$ with opposite $\operatorname{sign}^{42}$. The distribution of biexciton states follows the case near thermal equilibrium, which is the reason behind the inequivalent circularly co-polarised emission intensity under $\sigma^{+}$or $\sigma^{-}$, as shown in Fig. 3.

\section{Discussion}

We have discovered the quinton, the five-particle negatively charged biexciton in $1 \mathrm{~L}-\mathrm{WSe}_{2}$, unambiguously, as well as the neutral biexciton and its fine structure. Immediate next steps include the unequivocal verification of the $\mathrm{X}^{--}$state and the identification of bound states within the lower-energy peaks. A complete understanding of multi-exciton complexes is key to study coherent many-body phenomena, such as condensation ${ }^{7,8}$ and superfluidity ${ }^{9}$. Further, the ability to access and manipulate biexciton complexes in TMD-based heterostructures offers new routes towards probing other fundamental excitations in this system and the interplay between free and localised excitons. Extending our findings to the quantum confined regime will open new capabilities for cascaded emission of entangled photons and spin-multiphoton interfaces.

\section{Methods}

Sample fabrication and room-temperature characterisation. Bulk WSe $\mathrm{W}_{2}$ crystals are prepared by the flux zone growth method (see Supplementary Note 1). Bulk hBN crystals are grown by the temperature-gradient method under high pressure and high temperature. Graphite is sourced from NGS Naturgrafit. All bulk crystals are exfoliated by micromechanical cleavage ${ }^{43}$ on $\mathrm{Si} / \mathrm{SiO}_{2}$ (oxide thickness $285 \mathrm{~nm}$ ). $1 \mathrm{~L}$ - and FL-samples are identified by optical contrast ${ }^{44}$. Selected crystals are assembled within $\sim 5 \mathrm{~h}$ into LMHs via dry-transfer ${ }^{13,34}$. The $\mathrm{LMH}$ sample used for power-dependent and magnetic field-dependent PL measurements is formed, from top to bottom, of ML-hBN flakes ( $5 \mathrm{~nm}$ thick as determined by optical contrast), $1 \mathrm{~L}-\mathrm{WSe}_{2}$, and a second ML-hBN flake ( $\sim 10 \mathrm{~nm}$ thick as determined by optical contrast) and FLG ( $\sim$ layers thick as determined by optical contrast). That used for voltage-dependent measurements is prepared in a similar way, but the top ML$\mathrm{hBN}$ does not fully cover the $1 \mathrm{~L}-\mathrm{WSe}_{2}$ to allow for $\mathrm{Cr} / \mathrm{Au}(5 / 50 \mathrm{~nm})$ electrodes to 

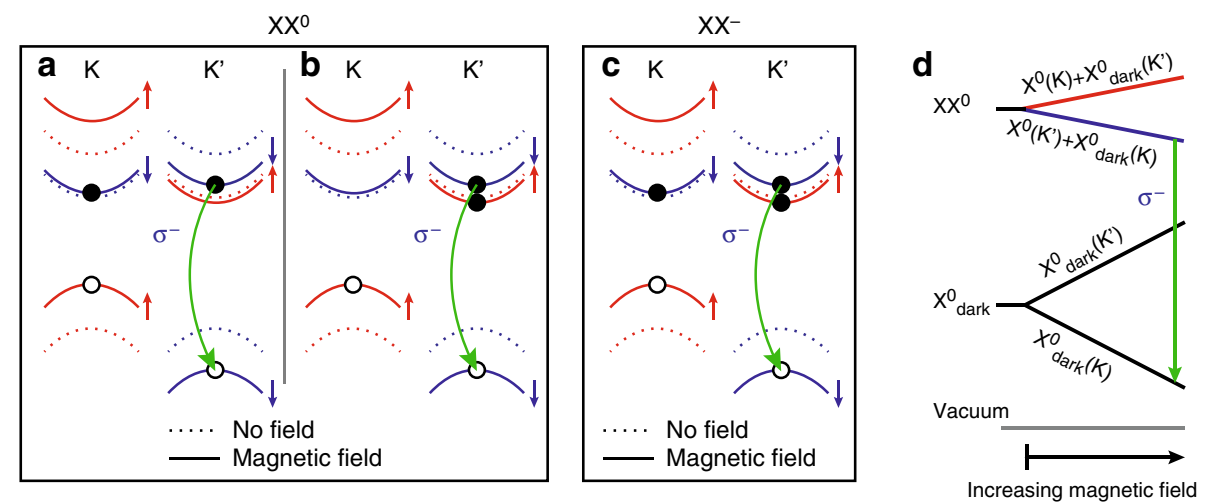

Fig. 4 Composition of biexciton species with applied magnetic field. $\mathbf{a}, \mathbf{b}, \mathbf{c}$ Single-particle picture of the internal structure of $(\mathbf{a}, \mathbf{b}) X X^{0}$ and $(\mathbf{c}) X X^{-}$for $B>$ 0 . The eigenstates shift inequivalently in $\mathrm{K}$ and $\mathrm{K}^{\prime}$ (dashed curves indicate no magnetic field, solid curves indicate applied magnetic field, red and blue colours indicate opposite spin). $X X^{0}$ comprises a bright exciton with highest radiative energy and a dark exciton with the electron (a) inter- or (b) intravalley with the bright exciton. $\mathbf{d}$ Many-body picture of the magnetic field effect on $X X^{0}$, comprising a bright and a dark exciton. Applying a magnetic field shifts the energy of the dark exciton more than that of $X X^{0}$ due to the higher $g$ of the former. This results in the dissociation of the biexciton in the form $\mathrm{XX}^{0} \rightarrow \mathrm{X}^{0}$ dark $+\gamma\left(\sigma^{-}\right)$, where $\gamma\left(\sigma^{-}\right)$is a photon with $\sigma^{-}$helicity

directly contact it. The second electrode contacts FLG. The electrodes are patterned by e-beam lithography followed by lift-off. The ML-hBN thickness is chosen to isolate the $1 \mathrm{~L}-\mathrm{WSe}_{2}$ from the environment, smoothen the roughness of $\mathrm{SiO}_{2}$, shield the charge-traps of the substrate and avoid tunnelling between FLG and $1 \mathrm{~L}-\mathrm{WSe}_{2}$, while not compromising the optical contrast under the optical microscope. Raman spectroscopy (Supplementary Fig. 1) and PL (Supplementary Fig. 2) are performed on the bulk crystals and after the assembly of LMH to characterise the starting material and confirm the $1 \mathrm{~L}-\mathrm{WS} e_{2}$ thickness ${ }^{45-47}$. Raman and PL spectra are acquired at room temperature using a Horiba LabRam Evolution (spectral resolution $\sim 0.3 \mathrm{~cm}^{-1}$ ) at $514.5 \mathrm{~nm}$. See Supplementary Note 2 for details on the roomtemperature optical characterisation.

Optical measurements at $\mathbf{4 K}$. Power dependent and gate-controlled measurements are performed in a variable-temperature Helium flow cryostat (Oxford Instruments Microstat HiRes2) with a home-built confocal microscope at a nominal temperature of $4.2 \mathrm{~K}$. The magneto-optical measurements are performed in a close-cycle bath cryostat (Attocube Attodry 1000) equipped with a superconducting magnet (maximum out-of-plane magnetic field $8 \mathrm{~T}$ ) at a nominal sample temperature of $3.8 \mathrm{~K}$. In the main text we refer to measurements at $4 \mathrm{~K}$ as an average of these two nominal temperatures.

Theoretical calculations. We use Mott-Wannier model and quantum Monte Carlo (QMC) as implemented in CASINO ${ }^{48}$ to calculate the energies of $\mathrm{X}^{0}, \mathrm{XX}^{0}$ and $\mathrm{XX}^{-}$in ML-WSe ${ }_{2}{ }^{29}$. The full photoemission spectra of $\mathrm{ML}^{-} \mathrm{WSe}_{2}$ in vacuum are reported in ref. ${ }^{29}$. To consider the effect of the dielectric screening provided by $\mathrm{hBN}$, we use the experimental value of the binding energy of $\mathrm{XX}^{0}$ and use Eq. (48) of ref. ${ }^{29}$ to derive the screening parameter $r^{*}$ which is $54 \AA$. We use the many-body GW electron and hole effective masses as $0.29 m_{0}$ and $0.34 m_{0}{ }^{49}$, respectively, where $m_{0}$ is the bare electron mass. We calculate the binding energy of $\mathrm{XX}^{-}$by subtracting the total energy of the exciton and trion from the total energy of $\mathrm{XX}^{-}$.

Data availability. The datasets generated and analysed during the current study are available from the corresponding author on reasonable request.

Received: 23 May 2018 Accepted: 13 July 2018

Published online: 13 September 2018

\section{References}

1. Chernikov, A. et al. Exciton binding energy and nonhydrogenic Rydberg series in monolayer $\mathrm{WS}_{2}$. Phys. Rev. Lett. 113, 076802 (2014).

2. Wang, G. et al. Colloquium: excitons in atomically thin transition metal dichalcogenides. Rev. Mod. Phys. 90, 021001 (2018).

3. Xiao, D., Liu, G.-B., Feng, W., Xu, X. \& Yao, W. Coupled spin and valley physics in monolayers of MoS2 and other Group-VI dichalcogenides. Phys. Rev. Lett. 108, 196802 (2012).

4. Mak, K. F., He, K., Shan, J. \& Heinz, T. F. Control of valley polarization in monolayer $\mathrm{MoS}_{2}$ by optical helicity. Nat. Nanotechnol. 7, 494-498 (2012).
5. Zeng, H., Dai, J., Yao, W., Xiao, D. \& Cui, X. Valley polarization in $\mathrm{MoS}_{2}$ monolayers by optical pumping. Nat. Nanotechnol. 7, 490-493 (2012).

6. Sidler, M. et al. Fermi polaron-polaritons in charge-tunable atomically thin semiconductors. Nat. Phys. 13, 255-261 (2017).

7. Kogar, A. et al. Signatures of exciton condensation in a transition metal dichalcogenide. Science 358, 1314-1317 (2017).

8. Cotlet, O., Zeytinoğlu, S., Sigrist, M., Demler, E. \& Imamoğlu, A. Superconductivity and other collective phenomena in a hybrid Bose-Fermi mixture formed by a polariton condensate and an electron system in two dimensions. Phys. Rev. B 93, 054510 (2016).

9. Fogler, M. M., Butov, L. V. \& Novoselov, K. S. High-temperature superfluidity with indirect excitons in van der Waals heterostructures. Nat. Commun. 5, 4555 (2014)

10. Jones, A. M. et al. Optical generation of excitonic valley coherence in monolayer $\mathrm{WSe}_{2}$. Nat. Nanotechnol. 8, 634-638 (2013).

11. Palacios-Berraquero, C. et al. Large-scale quantum-emitter arrays in atomically thin semiconductors. Nat. Commun. 8, 15093 (2017).

12. Branny, A., Kumar, S., Proux, R. \& Gerardot, B. D. Deterministic straininduced arrays of quantum emitters in a two-dimensional semiconductor Nat. Commun. 8, 15053 (2017).

13. Palacios-Berraquero, C. et al. Atomically thin quantum light-emitting diodes. Nat. Commun. 7, 12978 (2016).

14. Mak, K. F., Lee, C., Hone, J., Shan, J. \& Heinz, T. F. Atomically thin $\mathrm{MoS}_{2}$ : a new direct-gap semiconductor. Phys. Rev. Lett. 105, 136805 (2010).

15. Splendiani, A. et al. Emerging photoluminescence in monolayer $\mathrm{MoS}_{2}$. Nano. Lett. 10, 1271-1275 (2010).

16. Mak, K. F. et al. Tightly bound trions in monolayer $\mathrm{MoS}_{2}$. Nat. Mater. 12, 207-211 (2013).

17. Ross, J. S. et al. Electrical control of neutral and charged excitons in a monolayer semiconductor. Nat. Commun. 4, 1474 (2013).

18. Gourley, P. L. \& Wolfe, J. P. Experimental determination of equilibrium constants for excitonic systems in stressed, ultrapure silicon. Phys. Rev. B 25, 6338-6348 (1982).

19. Kim, J. C., Wake, D. R. \& Wolfe, J. P. Thermodynamics of biexcitons in a GaAs quantum well. Phys. Rev. B 50, 15099-15107 (1994)

20. You, Y. et al. Observation of biexcitons in monolayer $\mathrm{WSe}_{2}$. Nat. Phys. 11, 477-481 (2015).

21. Dey, P. et al. Biexciton formation and exciton coherent coupling in layered GaSe. J. Chem. Phys. 142, 212422 (2015).

22. Plechinger Gerd et al. Identification of excitons, trions and biexcitons in single-layer $\mathrm{WS}_{2}$. Phys. Status Solidi - RRL 9, 457-461 (2015).

23. Sie, E. J., Frenzel, A. J., Lee, Y.-H., Kong, J. \& Gedik, N. Intervalley biexcitons and many-body effects in monolayer $\mathrm{MoS}_{2}$. Phys. Rev. B 92, 125417 (2015).

24. Hao, K. et al. Neutral and charged inter-valley biexcitons in monolayer $\mathrm{MoSe}_{2}$ Nat. Commun. 8, 15552 (2017).

25. Nagler, P. et al. Zeeman splitting and inverted polarization of biexciton emission in monolayer $\mathrm{WS}_{2}$. Preprint at http://arXiv.org/abs/1801.09255 (2018).

26. Paradisanos, I. et al. Room temperature observation of biexcitons in exfoliated $\mathrm{WS}_{2}$ monolayers. Appl. Phys. Lett. 110, 193102 (2017).

27. Cadiz, F. et al. Excitonic linewidth approaching the homogeneous limit in $\mathrm{MoS}_{2}$-Based van der Waals heterostructures. Phys. Rev. X 7, 021026 (2017). 
28. Tongay, S. et al. Defects activated photoluminescence in two-dimensional semiconductors: interplay between bound, charged, and free excitons. Sci. Rep. 3, 2657 (2013)

29. Mostaani, E. et al. Diffusion quantum Monte Carlo study of excitonic complexes in two-dimensional transition-metal dichalcogenides. Phys. Rev. B 96, 075431 (2017).

30. Szyniszewski, M., Mostaani, E., Drummond, N. D. \& Fal'ko, V. I. Binding energies of trions and biexcitons in two-dimensional semiconductors from diffusion quantum Monte Carlo calculations. Phys. Rev. B 95, 081301 (2017).

31. Kidd, D. W., Zhang, D. K. \& Varga, K. Binding energies and structures of twodimensional excitonic complexes in transition metal dichalcogenides. Phys. Rev. B 93, 125423 (2016)

32. Mayers, M. Z., Berkelbach, T. C., Hybertsen, M. S. \& Reichman, D. R. Binding energies and spatial structures of small carrier complexes in monolayer transition-metal dichalcogenides via diffusion Monte Carlo. Phys. Rev. B 92, 161404 (2015).

33. Van der Donck, M., Zarenia, M. \& Peeters, F. M. Excitons, trions, and biexcitons in transition-metal dichalcogenides: magnetic-field dependence. Phys. Rev. B 97, 195408 (2018).

34. Zomer, P. J., Guimarães, M. H. D., Brant, J. C., Tombros, N. \& van Wees, B. J. Fast pick up technique for high quality heterostructures of bilayer graphene and hexagonal boron nitride. Appl. Phys. Lett. 105, 013101 (2014).

35. Courtade, E. et al. Charged excitons in monolayer $\mathrm{WSe}_{2}$ : experiment and theory. Phys. Rev. B 96, 085302 (2017).

36. Wang, G. et al. In-plane propagation of light in transition metal dichalcogenide monolayers: optical selection rules. Phys. Rev. Lett. 119, 047401 (2017).

37. Zhou, Y. et al. Probing dark excitons in atomically thin semiconductors via near-field coupling to surface plasmon polaritons. Nat. Nanotechnol. 12, 856-860 (2017).

38. Plechinger, G. et al. Trion fine structure and coupled spin-valley dynamics in monolayer tungsten disulfide. Nat. Commun. 7, 12715 (2016).

39. Jones, A. M. et al. Excitonic luminescence upconversion in a two-dimensional semiconductor. Nat. Phys. 12, 323 (2016).

40. Aivazian, G. et al. Magnetic control of valley pseudospin in monolayer $\mathrm{WSe}_{2}$. Nat. Phys. 11, 148-152 (2015)

41. Srivastava, A. et al. Valley Zeeman effect in elementary optical excitations of monolayer $\mathrm{WSe}_{2}$. Nat. Phys. 11, 141-147 (2015).

42. Robert, C. et al. Fine structure and lifetime of dark excitons in transition metal dichalcogenide monolayers. Phys. Rev. B 96, 155423 (2017).

43. Novoselov, K. S. et al. Two-dimensional atomic crystals. PNAS 102, 10451-10453 (2005).

44. Casiraghi, C. et al. Rayleigh imaging of graphene and graphene layers. Nano. Lett. 7, 2711-2717 (2007).

45. Zhao, W. et al. Lattice dynamics in mono- and few-layer sheets of $\mathrm{WS}_{2}$ and $\mathrm{WSe}_{2}$. Nanoscale 5, 9677-9683 (2013).

46. Zhao, W. et al. Evolution of electronic structure in atomically thin sheets of $\mathrm{WS}_{2}$ and $\mathrm{WSe}_{2} .7,791-797$ (2012).

47. Terrones, H. et al. New first order raman-active modes in few layered transition metal dichalcogenides. Sci. Rep. 4, 4215 (2014).

48. Needs, R. J., Towler, M. D., Drummond, N. D. \& Ríos, P. L. Continuum variational and diffusion quantum Monte Carlo calculations. J. Phys. Condens. Matter 22, 023201 (2010).
49. Shi, H., Pan, H., Zhang, Y.-W. \& Yakobson, B. I. Quasiparticle band structures and optical properties of strained monolayer $\mathrm{MoS}_{2}$ and $\mathrm{WS}_{2}$. Phys. Rev. B 87, 155304 (2013).

\section{Acknowledgements}

The authors thank Bernhard Urbaszek, Neil D. Drummond, Vladimir I. Fal'ko and Ioannis Paradisanos for useful discussions. We acknowledge funding from NSF DMR1552220, Elemental Strategy Initiative conducted by the MEXT, Japan and the CREST (JPMJCR15F3), JST, EU Graphene Flagship, ERC Grants Hetero2D and PHOENICS, EPSRC Grants EP/509K01711X/1, EP/K017144/1, EP/N010345/1, EP/M507799/ 5101, and EP/L016087/1, Marie Skłodowska-Curie Actions Spin-NANO, Grant No. 676108, Quantum Technology Hub NQIT EP/M013243/1.

\section{Author contributions}

M.B., A.C.F. and M.A. conceived and managed the project; K.W. and T.T. provided hBN

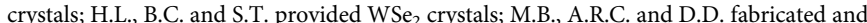
characterised the devices; M.B., A.R.-P.M., D.M.K., C.P.-B., B.P. and M.A. performed the low-temperature PL measurements and analysed the results; E.M. performed the calculations. All authors participated in the discussion of the results and the writing of the manuscript.

\section{Additional information}

Supplementary Information accompanies this paper at https://doi.org/10.1038/s41467 018-05632-4.

Competing interests: The authors declare no competing interests.

Reprints and permission information is available online at http://npg.nature.com/ reprintsandpermissions/

Publisher's note: Springer Nature remains neutral with regard to jurisdictional claims in published maps and institutional affiliations.

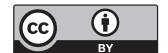

Open Access This article is licensed under a Creative Commons Attribution 4.0 International License, which permits use, sharing, adaptation, distribution and reproduction in any medium or format, as long as you give appropriate credit to the original author(s) and the source, provide a link to the Creative Commons license, and indicate if changes were made. The images or other third party material in this article are included in the article's Creative Commons license, unless indicated otherwise in a credit line to the material. If material is not included in the article's Creative Commons license and your intended use is not permitted by statutory regulation or exceeds the permitted use, you will need to obtain permission directly from the copyright holder. To view a copy of this license, visit http://creativecommons.org/ licenses/by/4.0/.

(C) The Author(s) 2018 